\title{
Pregnancy Time Period
}

National Cancer Institute

\section{Source}

National Cancer Institute. Pregnancy Time Period. NCI Thesaurus. Code C92858.

The duration of pregnancy from conception to childbirth. 